\title{
A Note on the Generalized Effects of Assortative Mating
}

\author{
L. J. Eaves, ${ }^{1}$ A. C. Heath, ${ }^{1}$ and N. G. Martin ${ }^{1}$
}

Received 1 Mar. 1984-Final 27 Apr. 1984

Several twin studies of multiple abilities and educational and socioeconomic variables suggest that a single common factor underlies the contribution of mate selection and cultural inheritance to the covariation of such measures. Theoretical analysis shows that this finding is consistent with a mechanism of mate selection in which specific abilities are merely components of a composite latent variable for which there is assortative mating. We question how far measures of specific abilities reflect behavioral traits of independent adaptive significance.

KEY WORDS: assortative mating; cultural inheritance; trait correlation.

\section{INTRODUCTION}

It is well known that specific ability measures are correlated. Over the last 80 years several models have been proposed for the phenotypic covariation between abilities, ranging from the common factor model of Spearman (1927), which postulates a single factor common to all measures, to the multiple group factor models of such pioneers as Thurstone (1938). Without exception, these workers focused on the phenotypic correlations between measures at the expense of analyzing their causal basis in terms of genetic and environmental factors.

Several investigators (e.g., Loehlin and Vandenberg, 1968; Bock and Vandenberg, 1968; Eaves and Gale, 1974; Plomin and DeFries, 1979) have tried comparatively primitive approaches to estimating genetic and

This work was supported by grants GM30250 and HL28922 from the National Institutes of Health.

${ }^{1}$ Department of Human Genetics, Medical College of Virginia, Richmond, Virginia 23298. 
environmental components of trait covariation but have used inadequate methods (see discussion by Martin and Eaves, 1977).

Although it has been recognized that assortative mating can generate correlations between measures (e.g., Thompson, 1967), the implications of assortative mating for specific abilities have scarcely been appreciated. In this paper we outline a model which predicts a generalized effect of assortative mating on specific abilities and socioeconomic variables. We argue that published multivariate analyses of specific abilities are consistent with the hypothesis that genetic effects on each ability also contribute, directly or indirectly, to a composite latent variable for which assortative mating occurs.

\section{BACKGROUND}

If we assume additivity of genes and environment, then the phenotypic covariance $\mathbf{P}$ of multiple measures may be decomposed into three independent components:

$$
\mathbf{P}=\mathbf{G}+\mathbf{E}+\mathbf{B} .
$$

Here $\mathbf{G}$ is the matrix of additive genetic variances and covariances, $\mathbf{E}$ is the within-family environmental covariance matrix, and $\mathbf{B}$ is the covariance matrix due to the combined effects of cultural inheritance and assortative mating (Martin and Eaves, 1977). Maximum-likelihood methods may be employed to estimate $\mathbf{G}, \mathbf{E}$, and $\mathbf{B}$ or their factors, for multiple measures on samples of monozygotic (MZ) and dizygotic (DZ) twins (Martin and Eaves, 1977; Fulker, 1978; Behrman et al., 1980).

Three studies have shown that $\mathbf{B}$ makes a highly significant contribution to family resemblance for multiple abilities (Martin and Eaves, 1977; Martin et al., 1984) and measures of educational and socioeconomic success (Fulker, 1978). Thus, it must be concluded that assortative mating and/or cultural inheritance make a substantial contribution to twin resemblance for such socially important variables.

A striking finding of these three studies is that $\mathbf{B}$ is consistently of rank 1 . This tells us that there is only a single common factor accounting for variation and covariation due to this source so that correlations between variables are all close to one insofar as they are caused by assortative mating or cultural transmission. On the other hand, $\mathbf{G}$ and $\mathbf{E}$ may be represented adequately only in terms of multiple factors and specific variances. In this note we consider a model for the contributions of assortative mating to $\mathbf{B}$ and how this might explain why the matrix has unit rank. We suggest that the model and the empirical data have im- 
portant implications for our understanding of the effect of mate selection on covariation between multiple variables.

\section{THE MODEL}

We assume that genetic variation in a set of multiple measures, $V_{1} \cdots V_{p}$, is caused by differences in a number of standardized latent genetic variables, $G_{1} \cdots G_{k}$. We let $h_{i j}$ be the regression of $V_{j}$ on $G_{i}$ and a typical element of the $p \times k$ matrix $\mathbf{H}$. $\mathbf{H}$ may be of any rank. The empirical data on multiple measures of twins show clearly that $\mathbf{H}$ is of rank $>1$ for abilities and socioeconomic variables.

Now the additional contribution of assortative mating to the crosssib covariances between multiple measures is

$$
\mathbf{B}=\mathbf{H A H}^{\prime} \text {. }
$$

A typical off-diagonal element $\alpha_{i j}(i \neq j)$ of $\mathbf{A}$ is the correlation between $G_{i}$ of one sibling (or DZ twin) and $G_{j}$ of the other. These correlations are assumed to arise by assortative mating alone.

The correlation between sibs for the same genetic component $\left(G_{i}\right.$, say) is $\gamma_{i i}$, which may be written $\gamma_{i i}=\frac{1}{2}+\alpha_{i i} / 2$, the term $\alpha_{i i} / 2$ resulting from assortative mating, and the remaining $\frac{1}{2}$ persists even under random mating. The diagonal elements of $\mathbf{A}$ are the $\alpha_{i i}$, not the $\gamma_{i i}$, since we are concerned only with additional variation and covaration due to assortative mating.

Since the empirical data already suggest that $\mathbf{H}$ is of rank $>1$, we can secure $\mathbf{B}$ of unit rank only if $\mathbf{A}$ is of unit rank. We show that this will be true if assortative mating is based on some linear combination of the measured variables or their underlying genetic determinants.

Figure 1 gives the elements of a model for the correlation between two typical genetic components $G_{i}$ and $G_{j}$. We assume that each of the genetic systems $G_{i} \cdots G_{k}$ contributes to a composite phenotype, $P$ ("general intelligence," "educational attainment," or "socioeconomic success"), on which mate selection is based. The contribution is measured by the path $h^{\prime}{ }_{i}$. The correlation, $\alpha^{\prime}{ }_{i j}$ between $G_{i}$ and $G_{j}$ in parents is given a "prime" to distinguish it from the corresponding correlation in the next generation. It is not necessary that $\alpha_{i j}=\alpha^{\prime}{ }_{i j}$ to obtain our result, although the constraint will hold for all $\alpha_{i j}$ at equilibrium under assortative mating.

The copath $\mu$ measures the degree of assortative mating between the latent, composite phenotypes of spouses. Employing the rules of path analysis modified by Cloninger (1980) to simplify the derivation of correlations from complex path diagrams, we find 


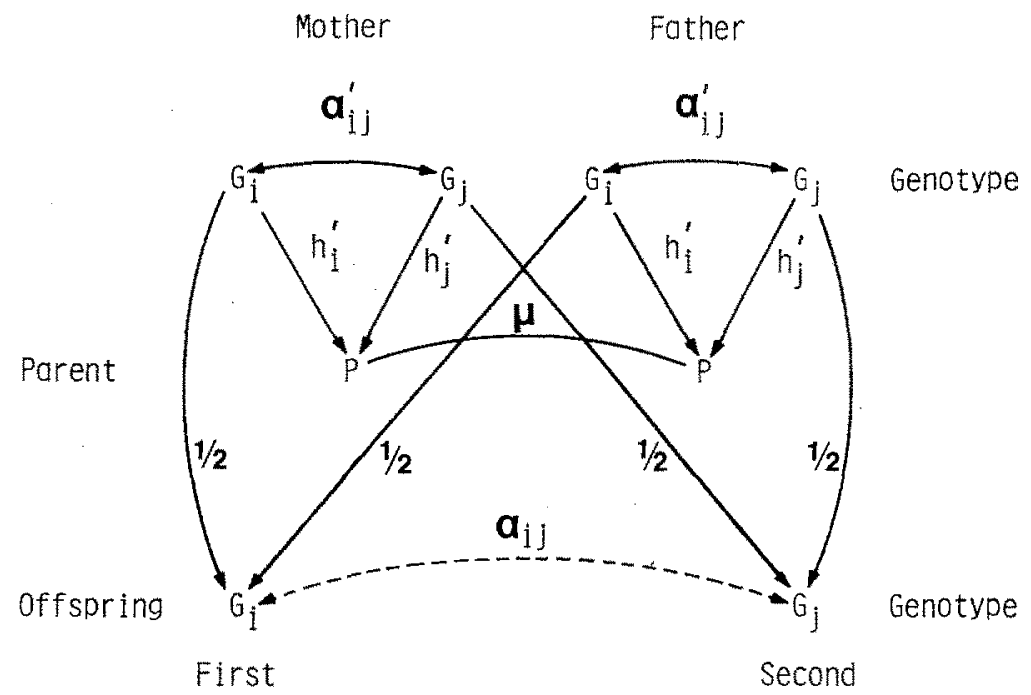

Fig. 1. Elements of a model for the generalized effects of assortative mating.

$$
\alpha_{i j}=\mu \rho_{i} \rho_{j} / 2,
$$

where $\rho_{i}$ is the correlation between $G_{i}$ and $P$. When $G$ comprises $k$ genetic variables, each consistent with the model in Fig. 1, we have

$$
\rho_{i}=h_{i}+\sum_{j=1}^{k} \alpha_{i j} h_{j}, \quad i \neq j .
$$

It is important to note that the $\alpha_{i j}$ are of the same form even if the $G$ 's do not contribute directly to $P$ as shown in Fig. 1. For example, the measured variables, $V_{1} \cdots V_{p}$, may intervene between the $G$ 's and $P$ or may be correlated with $P$ only through the $G$ 's. Although the $\rho_{i}$ 's will differ in these two circumstances, the $\alpha_{i j}$ 's will still be of the same form.

For $\mathbf{B}$ to have unit rank it is sufficient that $\mathbf{A}$ have unit rank. $\mathbf{A}$ will have rank 1 if all $2 \times 2$ determinants $\left|\mathbf{A}_{i j}\right|$ are zero. We put

$$
\mathbf{A}_{i j}=\left(\begin{array}{cc}
\alpha_{i i} & \alpha_{i j} \\
\alpha_{j i} & \alpha_{j j}
\end{array}\right)=\mu / 2\left(\begin{array}{cc}
\rho_{i}^{2} & \rho_{i} \rho_{j} \\
\rho_{j} \rho_{i} & \rho_{j}^{2}
\end{array}\right) .
$$

Since $\left|\mathbf{A}_{i j}\right|=\mathbf{0}$ for all $i, j$, then $\mathbf{A}$ and hence $\mathbf{B}$ have rank 1 .

\section{DISCUSSION}

Three studies of abilities and socioeconomic variables have shown that the genetic and within-family environmental covariance matrices ( $G$ 
and $\mathbf{E}$ ) have both common and specific factors. This is expected if only some of the genes have pleiotropic effects on more than one variable. The additional covariation due to assortative mating and cultural inheritance, $\mathbf{B}$, is of rank 1 .

Our model shows that this finding is consistent with assortative mating based on a single common latent variable to which each of the genetic factors underlying the measured variables contributes linearly and additively. The result will hold whatever the genetic relationship between the measured variables and the latent phenotype on which assortive mating is based. We recognize that twin data confound cultural effects and assortative mating. A comparable result will follow for cultural inheritance based on a single common latent variable.

Our result has important implications for the understanding of the biological significance of multiple ability tests and multiple indicators of socioeconomic success. The investment of psychologists in trying to break general abilities into specific components has been partially successful. At the genetic level, there is evidence that each test of a multiple ability battery is to some extent assessing the effects of different genes. However, when we consider how these component variables contribute to the biologically important decisions of mate selection and cultural inheritance, we are forced to acknowledge that neither do spouses recognize their specific components in selecting one another nor do educators recognize them in teaching children.

Mate selection and/or cultural inheritance exercise a more pervasive effect and operate through a general aspect of the phenotype. Such a general aspect of the phenotype is not necessarily measured any better by combining scores on multiple tests. It is possible that educational attainment may prove ultimately to be the major factor contributing to the joint effects of assortative mating and cultural inheritance on the correlation between multiple abilities.

One explanation may be that mate selection has been shaped by natural selection to be a "rational decision" process (Edwards, 1954). To make a rational choice between potential spouses varying in many different factors, humans must apply a consistent set of weights to these, using weighted scores on the derived latent dimension as the basis for choice. If these weights have evolved as population constants or differ only between sexes (Eaves and Heath, 1981), B would be predicted to be of rank 1. We predict that a joint analysis of abilities, education and socioeconomic variables, and other variables influencing choice of spouse will reveal significant communality based on a single latent dimension on which mate selection is based. 


\section{REFERENCES}

Behrman, J. R., Hrubec, Z., Taubman, P., and Wales, T. J. (1980). Socioeconomic Success. A Study of the Effects of Genetic Endowments, Family Environment and Schooling, North-Holland, New York.

Bock, R. D., and Vandenberg, S. G. (1968). Components of heritable variation in mental test scores. In Vandenberg, S. G. (ed.), Progress in Human Behavior Genetics, Johns Hopkins Press, Baltimore.

Cloninger, C. R. (1980). Interpretation of intrinsic and extrinsic structural relations by path analysis: Theory and applications to assortative mating. Genet. Res. 36:135-145.

Eaves, L. J., and Gale, J. S. (1974). A method for analysing the genetic basis of covariation. Behav. Genet. 4:253-267.

Eaves, L. J., and Heath, A. C. (1981). On the detection of asymmetric assortative mating. Nature 289:205-206.

Edwards, W. (1954). The theory of decision making. Psychol. Bull. 51:380-417.

Fulker, D. W. (1978). Multivariate extensions of a biometrical model of twin data. Twin Research: Psychology and Methodology, Alan R. Liss, New York, pp. 217-236.

Loehlin, J. C., and Vandenberg, S. G. (1968). Genetic and environmental components in the covariation of cognitive abilities: An additive model. In Vandenberg, S. G. (ed.), Progress in Human Behavior Genetics, Johns Hopkins Press, Baltimore.

Martin, N. G., and Eaves, L. J. (1977). The genetical analysis of covariance structure. Heredity 38:79-95.

Martin, N. G., Jardine, R., and Eaves, L. J. (1984). Is there only one set of genes for different abilities? A reanalysis of the National Merit Scholarship Qualifying Test (NMSQT) data. Behav. Genet. 14:355-370.

Plomin, R, and DeFries, J. C. (1979). Multivariate behavioral genetic analysis of twin data on scholastic abilities. Behav, Genet. 9:505-517.

Spearman, C. (1927). The Abilities of Man, Macmillan, London.

Thompson, W. R. (1966). Multivariate experiment in behavior genetics. In Cattell, R. B. (ed.), Handbook of Multivariate Experimental Psychology, Rand McNally, Chicago.

Thurstone, L. L. (1938). Primary Mental Abilities, University of Chicago Press, Chicago.

Edited by C. Robert Cloninger 